\title{
Implementación de una aplicación informática para la validación de artículos científicos en revistas indexadas
}

\section{Implementation of a computer application for the validation of scientific articles in indexed journals}

\begin{abstract}
Velasteguí López Luis Efraín ${ }^{1}$, Carrasco Ruano Yolanda Tatiana ${ }^{2}$, Fabara Villacis Manolo Roberto ${ }^{3} \&$. Diego Omar Guerra Poalasin ${ }^{4}$
\end{abstract}

\begin{abstract}
.
DOI: https://doi.org/10.33262/cienciadigital.v4i1.1059

Technology has evolved so rapidly that it is capable of making decisions with the use of mathematical equations, which has allowed the generation of new software that optimizes the daily problems of ordinary people in the beginning and academics today, as is the case of development of an application capable of applying the APA metrics and citation regulations as presented in the main objective of the project, substantially reducing the time of editing and review for both researchers and publishers, contributing to the strengthening of research through of the dissemination of it, which is the main mission of the Digital Science Network, linking with the policies of the National Development Plan, contributing to the increase in the percentage of investment in private sector $\mathrm{R} \& \mathrm{D}$ and contributing to requests for national patents when properly completing the books and research raised by the network. Technically the agile software development methodology (scrum) was used, in the administrative part it is intended to use a garage methodology. This project is focused on the national market at the beginning and internationally in subsequent phases by presenting an automated and validated form to be implemented in an indexed magazine in an agile and intelligent way.
\end{abstract}

\footnotetext{
${ }^{1}$ Ciencia Digital Editorial, Ambato, Ecuador, luisefrainvelastegui@cienciadigital.org

${ }^{2}$ Visionario Digital , Ambato, Ecuador, tatianacarrasco@cienciadigital.org

${ }^{3}$ Ciencia Digital, Ambato, Ecuador, manolofabara@cienciadigital.org

${ }^{4}$ Ciencia Digital Editorial, Ambato, Ecuador, diegoguerra@cienciadigital.org
} 
Keywords: Implementation, application, computing, validation, criteria, articles, indexed magazine

\section{Resumen.}

La tecnología ha evolucionado tan rápidamente que es capaz de tomar decisiones con el uso de ecuaciones matemáticas, lo que ha permitido la generación de nuevo software que optimiza los problemas cotidianos de gente común en un inicio y académicos en la actualidad, como es el caso del desarrollo de una aplicación capaz de aplicar las métricas y normativa de citación de la APA como se presenta en el objetivo principal del proyecto, reduciendo sustancialmente el tiempo de edición y revisión tanto para investigadores como para editores, contribuyendo con el fortalecimiento de la investigación a través de la difusión de la misma que es la misión principal de la Red Ciencia Digital, vinculándose con las políticas del Plan Nacional de Desarrollo, al contribuir con el incremento en el porcentaje de inversión en I+D del sector privado y contribuir a las solicitudes de patentes nacionales al culminar adecuadamente los libros e investigaciones planteadas por la red. Técnicamente se usó la metodología de desarrollo ágil de software (scrum), en la parte administrativa se pretende utilizar una metodología de garaje. El presente proyecto está enfocado en el mercado nacional en un inicio y al internacional en fases subsecuentes al presentar un formulario automatizado y validado para ser implementado en una revista indexada de manera ágil e inteligente.

Palabras claves: Implementación, aplicación, informático, validación, criterios, artículos, revista indexadas

\section{Introducción.}

Según (B., 2012) La imaginación del hombre no tiene límites conocidos y la inquietud del espíritu lo lleva a indagar. El simple contacto con el ambiente que lo rodea no es suficiente, sino que se siente impulsado a averiguar el significado y esencia de las cosas: que son los objetos y cuál es la razón de su existencia. Por consiguiente la investigación científica es el procedimiento ordenado a seguir para establecer lo significativo de los hechos fenómenos hacia los cuales se dirige el interés científico, y para hallar y enseñar lo que en materia de ciencia es verdad.

(Salinas, 1995) El principal objetivo de la metodología de la investigación es que las personas estén capacitadas para realizar estudios e investigaciones científicas, en forma lógica y ordenada. Algunos autores proponen como definición de la metodología de la investigación científica que es el estudio sistemático, controlado, reflexivo y crítico de proposiciones hipotéticas sobre las supuestas relaciones que existen entre fenómenos naturales, o que es el proceso sistemático, lógico y organizado para adquirir conocimientos y resolver problemas. 
La investigación científica es de vital importancia en el ámbito académico y laboral ya que por medio de este recurso se puede obtener una gran cantidad de información necesaria para realizar cualquier actividad que nos propongamos o intentemos elaborar, además con la ayudad de una metodología de investigación lograremos darle un enfoque más óptimo y sistemático lo que intentamos realizar y poner en práctica.

(Visbal, 1995) La unesco ha sentenciado que "la finalidad esencial de un artículo científico es comunicar los resultados de investigaciones, ideas y debates de una manera clara, concisa y fidedigna; la publicación es uno de los métodos inherentes al trabajo científico. Es preciso establecer estrategias de publicación bien elaboradas y seguir con conocimiento de causa una serie de normas adecuadas para facilitar el intercambio entre científicos de todos los países y reducir a proporciones razonables el incremento del volumen de publicaciones"

(Trainig, 2002) Una de las cuestiones más sorprendentes acerca de publicar en revistas científicas es que todo el mundo lo considera algo muy difícil. Y en muchos casos así es. La capacidad para escribir un artículo científico sólo se relaciona con la capacidad para escribir otro artículo científico. No se debe considerar como una medida de la capacidad clínica o científica, la inteligencia o el valor como ser humano. Los que consiguen publicar son aquellos que tienen la motivación suficiente (véase «segundo paso») y han resuelto con éxito todo el resto de pasos necesarios para conseguir publicar.

Mediante la elaboración de un artículo científico podemos dar a conocer los resultados obtenidos de una investigación científica, además permitirá estructurar de una manera fidedigna el contenido de una investigación lo cual le dará un añadido al trabajo realizado, lo cual será aceptado y refutado por cualquier revista digital o comunidad académica.

(Bejarano, 2008). El programa SciELO (Scientific Electronic Library Online) es una biblioteca virtual para América Latina, el Caribe, España y Portugal, en una red que opera en dos campos, las colecciones regionales con oficinas de operación en cada país y las áreas temáticas. SciELO-Colombia es una biblioteca electrónica que incluye una colección de revistas científicas colombianas seleccionadas mediante un Comité Consultor Nacional constituido por Colciencias, la Organización Panamericana de la Salud, la Universidad Nacional de Colombia y representantes de los editores. La biblioteca forma parte de un proyecto desarrollado por la Fundação de Amparo à Pesquisa do Estado de São Paulo (FAPESP) en colaboración con el Centro Latinoamericano y del Caribe de Información en Ciencias de la Salud (BIREME) para ofrecer acceso gratuito al texto completo de los artículos.

(Project, 2002) Open Journal Systems (OJS) es un sistema de gestión y publicación de revistas que ha sido desarrollado por Public Knowledge Project a través de sus esfuerzos financiados por el gobierno federal para expandir y mejorar el acceso a la investigación. OJS ayuda en todas las etapas del proceso de publicación arbitrado, desde los envíos hasta la 
publicación e indexación en línea. A través de sus sistemas de gestión, su indexación finamente detallada de la investigación y el contexto que proporciona para la investigación, OJS busca mejorar la calidad académica y pública de la investigación arbitrada.

Para varias revistas digitales el contar con un aplicativo informático, que le permita al usuario publicar y realizar sus artículos de una forma sencilla e intuitiva es fundamental en el mundo actual, la escasez de plataformas que permitan hacer esta actividades es notoria lo cual dificulta el libre desarrollo y realización de las revistas digital actualmente.

(Wordpress, 2000) Permite crear un sitio web que satisfaga las necesidades específicas de la. Además permite crear un blog, un sitio profesional, un portafolio, una tienda en línea o cualquier cosa que se pueda imaginar. Con una optimización integrada y temas adaptables para móviles, con la cual se puede llegar a tantos usuarios como se necesite.

El objetivo de la presente investigación fue; realizar la implementación de una aplicación informática para la validación de artículos científicos en revistas indexadas., que sirva de apoyo para la gestión y validación de artículos científicos en base a parámetros como; número de hojas, numero de palabras, bibliografía entre otros aspectos importantes al momento de realizar un artículo y publicarlo dentro de revistas digitales tanto internacionales como nacionales.

\section{Metodología}

La presente investigación se realizó bajo las siguientes modalidades:

Modalidad Bibliográfica o Documentada: La investigación fue bibliográfica porque permitió ampliar y profundizar diferentes enfoques, teorías, conceptualizaciones y criterios de diversos autores sobre el tema de estudio.

Además la investigación fue experimental porque se intentó buscar la mejor solución que se adapte a las necesidades y requisitos que exigía la presente investigación.

\section{Herramientas e instrumentos de desarrollo}

- Wordpress

Es un gestor de contenidos basados en la creación de blog o bitácoras web el cual está enfocado a la creación y personalización de una página web que permite un alojamiento gratuito o de pago.

- Docxpresso

Es una biblioteca de software escrita en PHP que ha sido diseñado para generar documentos e informes basados en datos en un servidor web. Docxpresso es mucho más que una biblioteca que lo ayuda a transformar el código HTML5 en documentos PDF. (Docxpresso, 2018)

- PHP (acrónimo recursivo de PHP: Hypertext Preprocessor) 
Es un lenguaje de código abierto muy popular especialmente adecuado para el desarrollo web y que puede ser incrustado en HTML. Lo que distingue a PHP de algo del lado del cliente como Javascript es que el código es ejecutado en el servidor, generando HTML y enviándolo al cliente. El cliente recibirá el resultado de ejecutar el script, aunque no se sabrá el código subyacente que era. El servidor web puede ser configurado incluso para que procese todos los ficheros HTML con PHP, por lo que no hay manera de que los usuarios puedan saber qué se tiene debajo de la manga. (Php, 2001)

\section{- JavaScript}

Es un lenguaje de programación que te permite realizar actividades complejas en una página web - cada vez más una página web hace más cosas que sólo mostrar información estática - como mostrar actualizaciones de contenido en el momento, interactuar con mapas, animaciones gráficas 2D/3D etc. — puedes estar seguro que JavaScript está involucrado. Es la tercera capa del pastel de los estándares en las tecnologías para la web, dos de las cuales son (HTML y CSS), hablaremos de ellas más adelante con más detalle en otra parte de nuestra Área de Aprendizaje. (Docs, 2019)

- CSS

Hojas de Estilo en Cascada (del inglés Cascading Style Sheets) o CSS es el lenguaje utilizado para describir la presentación de documentos HTML o XML, esto incluye varios lenguajes basados en XML como son XHTML o SVG. CSS describe como debe ser renderizado el elemento estructurado en pantalla, en papel, hablado o en otros medios. (Docs, Developer mozilla, 2019)

\section{Recolección de la Información}

Para la recolección de información se utilizó la técnica de investigación documental de tipo informativa, obteniendo información relevante de diferentes fuentes confiables, además de páginas de revistas indexadas y editoriales digitales, analizando y seleccionando la información de mayor relevancia la cual sirvió de apoyo para el desarrollo de esta investigación.

\section{Procesamiento y análisis de la información}

Para el, procesamiento y análisis de la información se aplicó los siguientes procedimientos

- Recolección de información sobre los procesos requeridos para la generación y validación de un artículo científico.

- Análisis de la información obtenida.

- Interpretación de la información obtenida

- Diseño de proceso

- Desarrollo del aplicativo web. 


\section{Desarrollo del proyecto}

- Recolección de requerimientos para el aplicativo web

- Diseño de la base de datos para la aplicación web

- Diseño de la interfaz del aplicativo web

- Desarrollo y programación del aplicativo web

- Pruebas de validación y funcionamiento del aplicativo.

\section{Resultados}

Para realizar el desarrollo del formulario de validación del artículo se utilizó la herramienta Docxpresso la cual permitió generar automáticamente un archivo de Word con todo el contenido del artículo científico.

Lo primero que se realizo es formatear la plantilla del formato del artículo científico en formato .odt como se muestra en la figura.

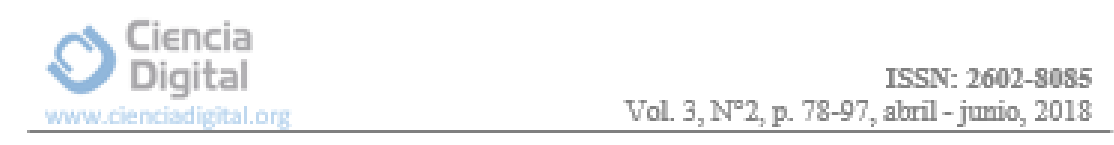

\section{$\{\{$ Titulo $\}\}$}

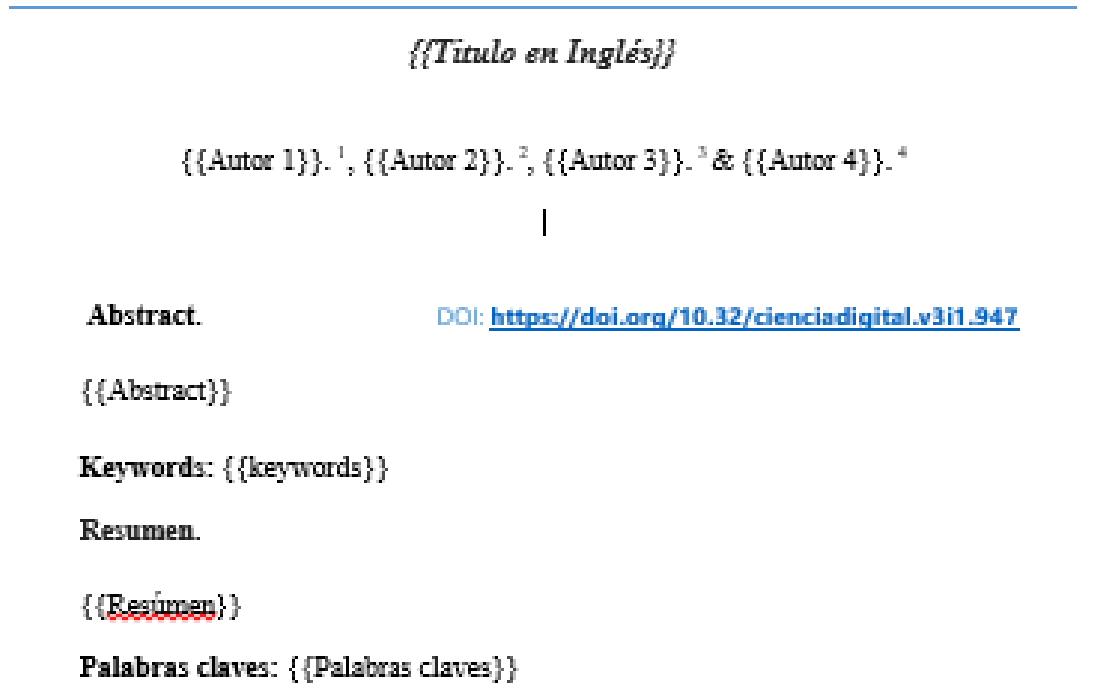

Figura 1; Plantilla formateada formato del articulo.

Elaborado por; Grupo de investigación. 
En cada sección del formato del artículo se procedió a colocar una variable entre llaves la cual recibirá toda la información prevista en cada sección del artículo título, abstract, resumen, etc

Por consiguiente el formato ya formateado en formato .odt se lo subió a la plataforma docxpresso, para posteriormente crear un formulario web con los campos requeridos para el formato del artículo científico. El formulario web quedo de la siguiente manera:

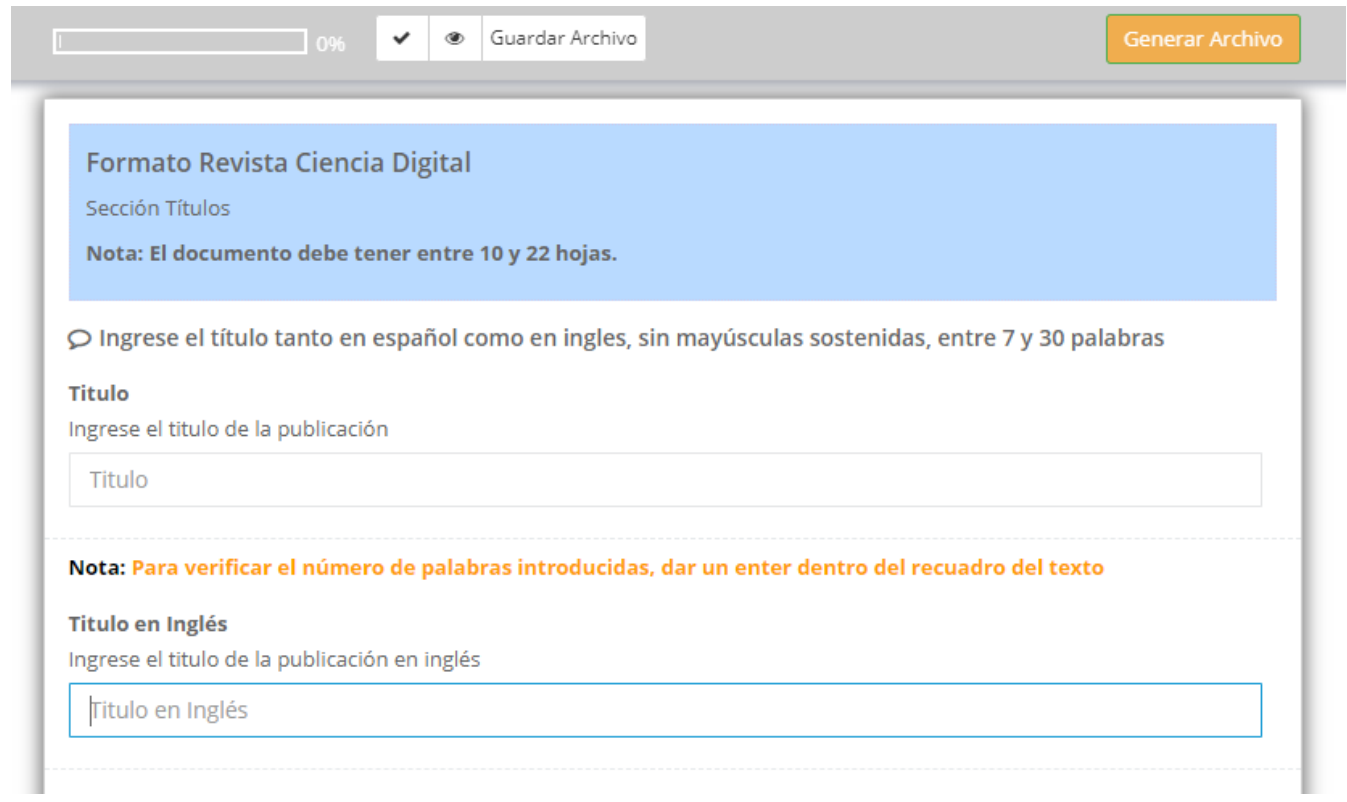

Figura 2; Formulario web/Sección Títulos

Elaborado por; Grupo de investigación.

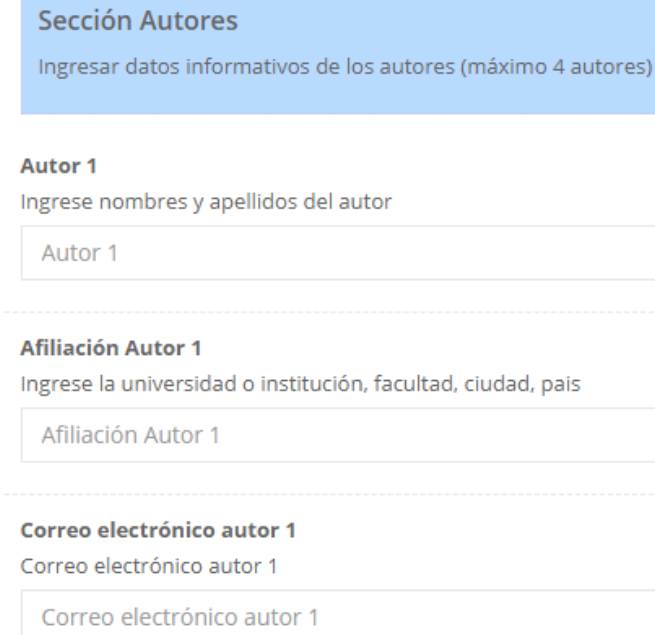

Figura 3; Formulario web/Sección Autores Elaborado por; Grupo de investigación. 


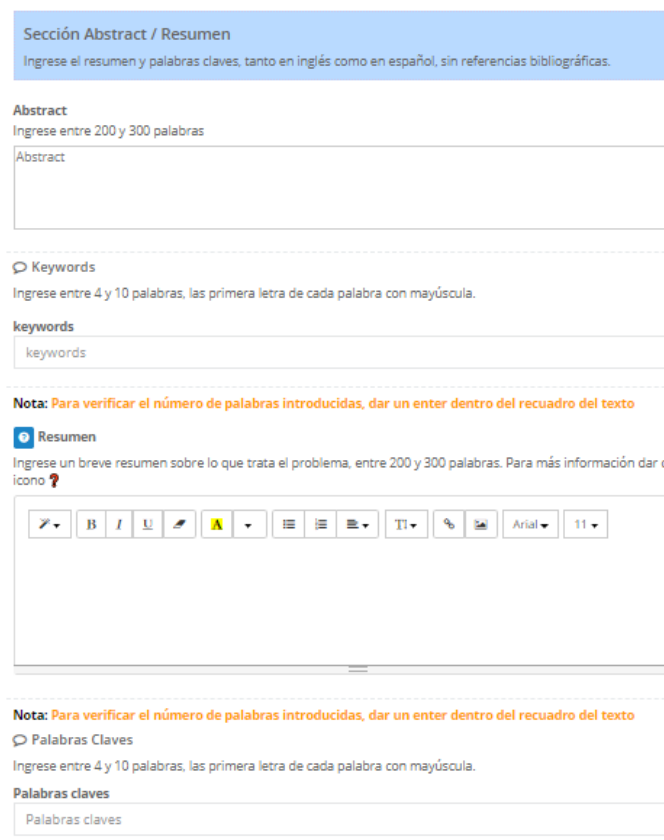

Figura 4; Formulario web/Sección Abstract - Resumen Elaborado por; Autores

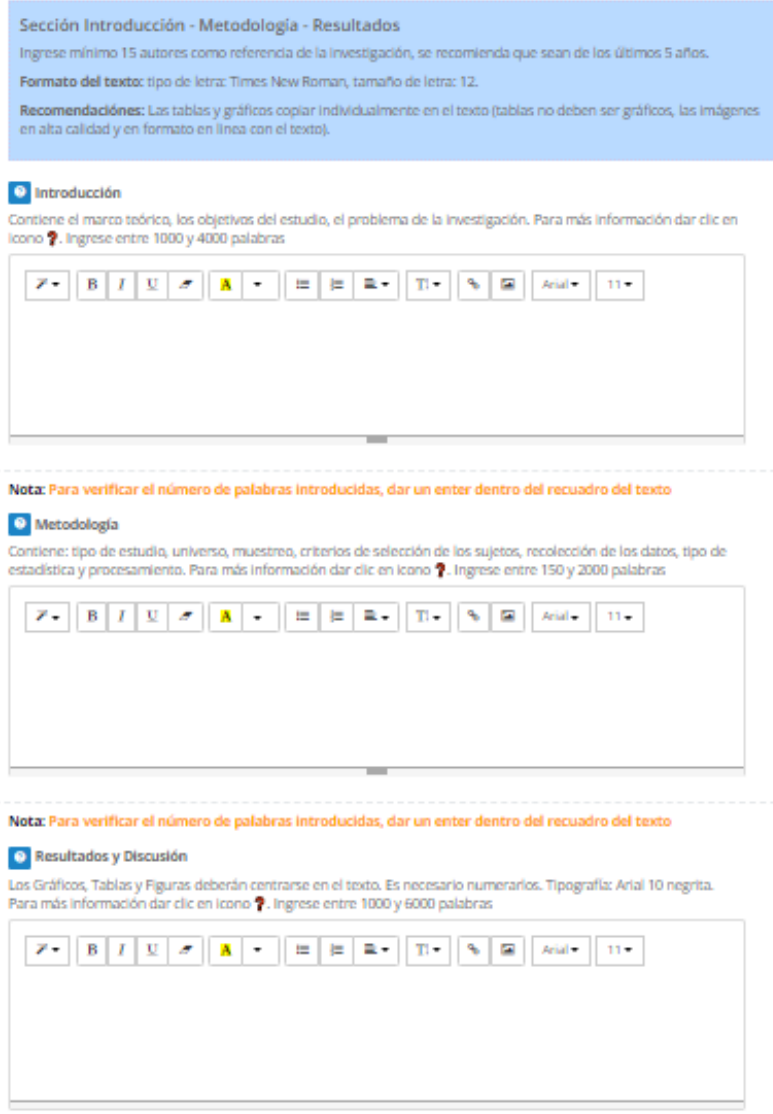

Figura 5; Formulario web/Sección Introducción - Metodología - Resultados Elaborado por; Grupo de investigación 


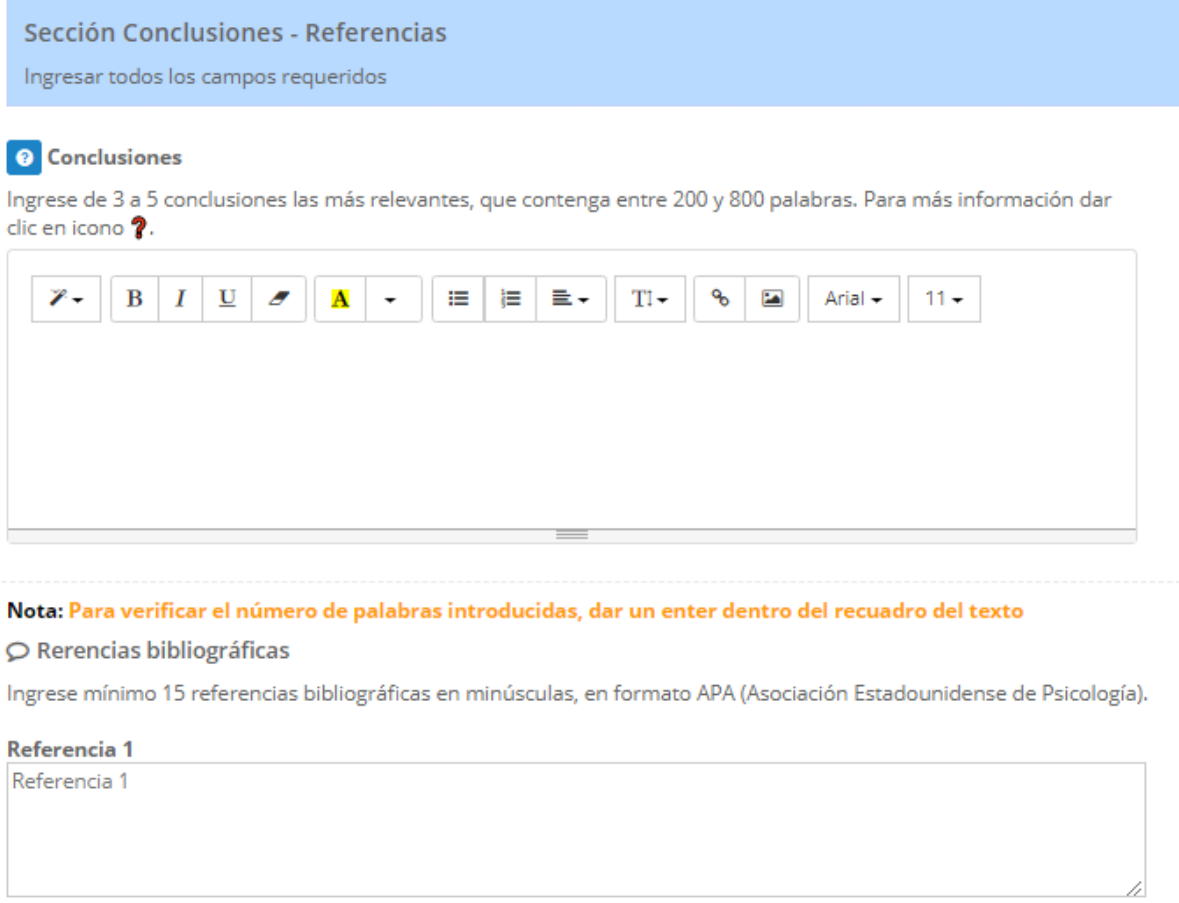

Figura 6; Formulario web/Sección Conclusiones - Bibliografías Elaborado por; Grupo de investigación.

En el formulario web se implementó el control respectivo en cada sección del artículo para controlar la cantidad de palabras y así normar el número de hojas previstas para la generación del artículo de la revista. El número de palabras por sección se las normo de la siguiente manera:

- Titulo (entre 7 y 10 palabras)

- Autores (máximo 4)

- Abstract/Resumen (entre 200 y 300 palabras)

- Keywords/Palabras claves (entre 4 y 10 palabras)

- Introducción (entre 1000 y 4000 palabras)

- Metodología (entre 150 y 2000 palabras)

- Resultados y Discusión (entre 1000 y 6000 palabras)

- Conclusiones (entre 3 y 5 conclusiones, de 200 a 800 palabras)

- Referencias bibliográficas (mínimo 10 referencias)

Para controlar estos lineamientos se procedió a programar el formulario web con código JavaScript a continuación un extracto del código realizado. 


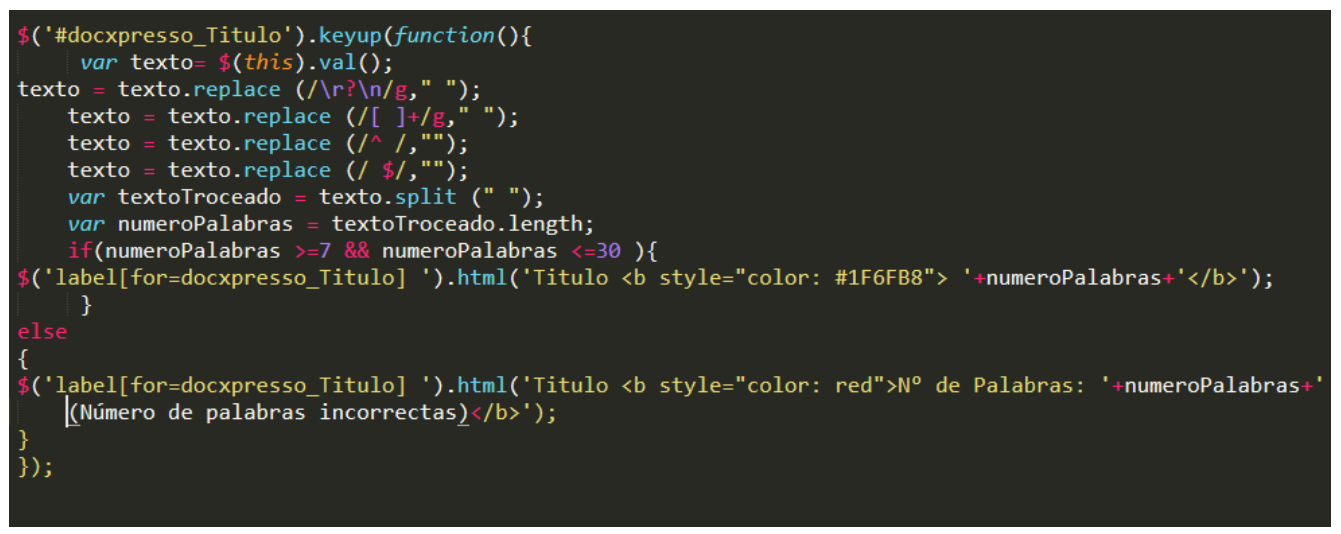

Figura 6; Código para control de palabras

Elaborado por; Grupo de investigación.

Al momento de generar el documento y no cumplir con los requerimientos anteriormente expuestos el formulario lanzara un mensaje de error como se puede observar en la imagen.

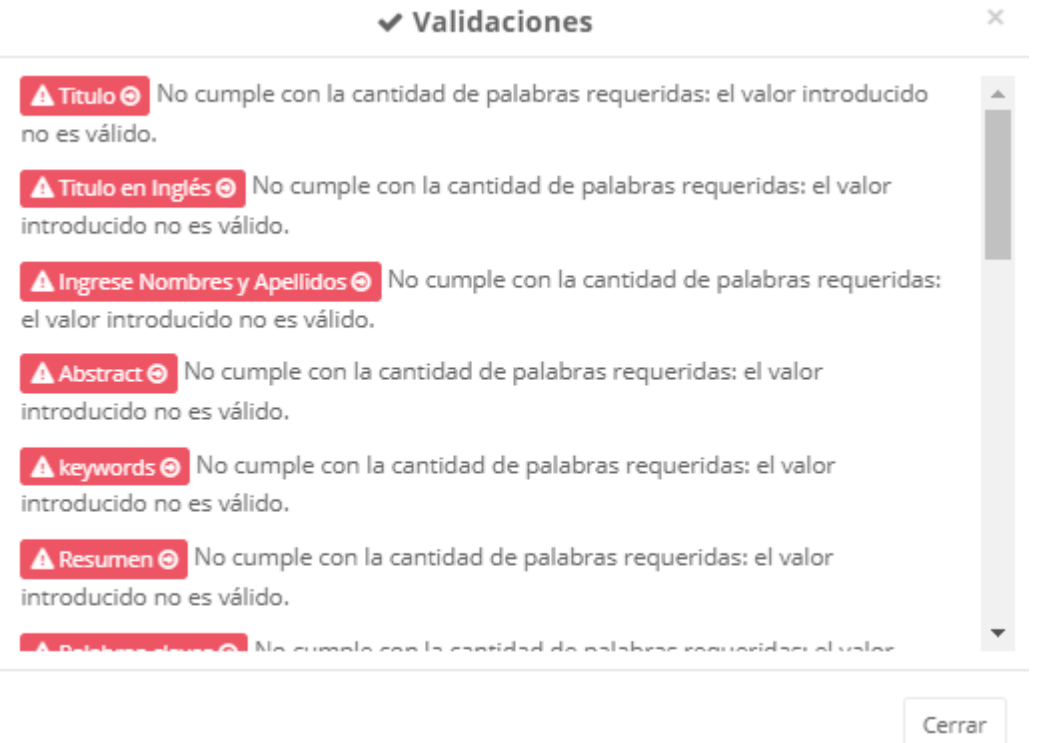

Figura 7; Mensajes de error formulario web

Elaborado por; Grupo de investigación.

Ingresados correctamente todo los datos en el formulario web se mostró un mensaje de opción de cómo se quiere generar el archivo del articulo sea como documento con extensión .doc o .docx. 


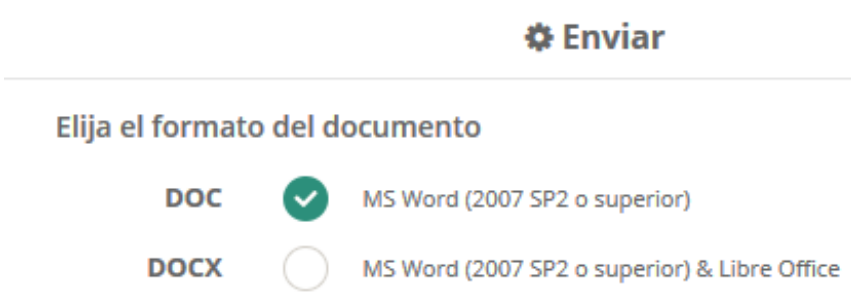

Figura 8; Opciones de generación de archivo Elaborado por; Grupo de investigación.

Después se generó un documento en Word que contiene toda la información del artículo académico a publicar el cual ya automáticamente se genera en el formato de la revista digital con todas las medidas, tamaños de letra y estilos que contenga.

Esta implementación del formulario web para la validación de artículos científicos es de vital importancia dentro del manejo de una revista digital ya que permitirá controlar diferentes lineamientos y requerimientos que pida la revista digital y así dar una mejorar calidad en la realización de documentos académicos e investigativos.

\section{Conclusiones.}

- Mediante la realización de esta investigación se obtuvo una solución óptima y viable para la solución y desarrollo del tema planteado, dando a conocer varios puntos esenciales al tener en cuenta al momento de realizar y publicar un artículo científico dentro de una revista digital indexada.

- El formulario web realizado permitió controlar todos los parámetros dentro de un documento, además se lo puede adaptar dentro de un aplicativo web o página web ya que presta varias herramientas para personalizarlo y adaptarlo a las necesidades de la revista digital.

\section{Referencias bibliográficas}

B., J. B. (2012). Que es la investigación científica. Antioquia: Revista Universidad EAFIT.

Bejarano, M. (2008). Indexación de las revistas. Revista Colombiana de Cirugía.

Docs, M. W. (11 de Mayo de 2019). Developer mozilla. Obtenido de https://developer.mozilla.org/es/docs/Learn/JavaScript/First_steps/Qu\%C3\%A9_es _JavaScript 
Docs, M. W. (10 de Septiembre de 2019). Developer mozilla. Obtenido de https://developer.mozilla.org/es/docs/Web/CSS

Docxpresso. (12 de Febrero de 2018). Docxpresso. Obtenido de https://www.docxpresso.com/

Php. (18 de Septiembre de 2001). Php. Obtenido de https://www.php.net/manual/es/introwhatis.php

Project, P. K. (22 de Enero de 2002). Obtenido de Public Knowledge Project : https://pkp.sfu.ca/ojs/

Salinas, P. J. (1995). METODOLOGÍA DE LA INVESTIGACIÓN CIENTÍFICA. Venezuela: Facultades de Ingeniería, Medicina, Odontología y Ciencias Forestales y Ambientales. Universidad de Los Andes.

Trainig, T. A. (2002). Cómo escribir artículos científicos fácilmente. New Zealand Journal of Medical Laboratory Science, 56.

Visbal, L. A. (1995). El artículo científico. Revista Cubana de Medicina General Integral, 150.

Wordpress. (2000). Obtenido de Wordpress.com: https://es.wordpress.com/

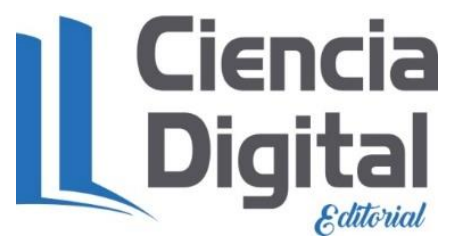




\section{PARA CITAR EL ARTÍCULO INDEXADO.}

Velasteguí López , E., Carrasco Ruano , Y. T., Fabara Villacis, M. R., \& Poalasin , D. O. (2020). Implementación de una aplicación informática para la validación de artículos científicos en revistas indexadas. Ciencia Digital, 4(1), 5-17. https://doi.org/10.33262/cienciadigital.v4i1.1059

\section{¿Ciencia}

El artículo que se publica es de exclusiva responsabilidad de los autores y no necesariamente reflejan el pensamiento de la Revista Ciencia Digital.

El artículo queda en propiedad de la revista y, por tanto, su publicación parcial y/o total en otro medio tiene que ser autorizado por el director de la Revista Ciencia Digital.
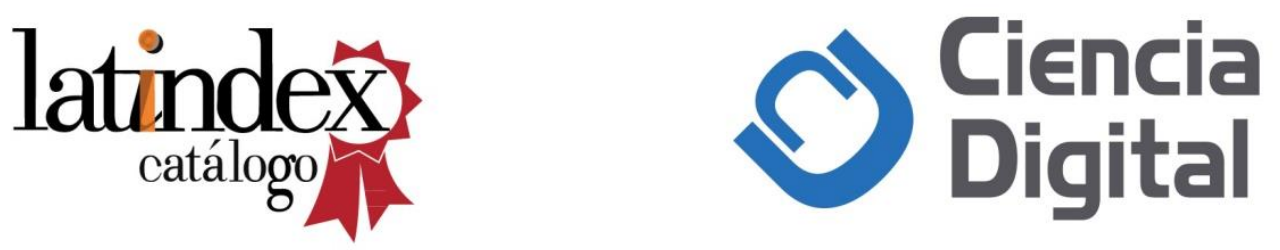